\title{
ESTUDO EXPERIMENTAL DE ZYGODONTOMYS LASIURUS (RODENTIA-CRICETIDAE) COM CEPAS DE TRYPANOSOMA CRUZI*
}

\author{
Monamaris $M$. Borges ** \\ Dalva A. Mello*** \\ Maria Lucia Teixeira **** \\ João D.B. da Silva *****
}

\begin{abstract}
BORGES, M. M. et al. Estudo experimental de zygodontomys lasiurus (Rodentia-cricetidae) com cepas de Trypanosoma cruzi. Rev. Saúde púbI., S. Paulo: 17:387-93, 1983.

RESUMO: São apresentados resultados em relação a infeç̧ão experimental de Zygodontomys lasiurus (Rodentia-Cricetidae) com duas cepas de T. cruzi isoladas de casos humanos, $Y$ e Berenice, e uma isolada de um triatomíneo silvestre, chamada costalimai. Foram realizados estudos em relação a evolução da parasitemia, duração da patência e prepatência da infecção. Com o objetivo de verificar a agressividade e tropismo tissular das cepas de $\mathbf{T}$. cruzi nesta espécie de roedor, foram também realizados estudos histopatológicos. Os resultados obtidos mostraram que os niveis de parasitemias foram baixos para as três cepas estudadas. A patência da infecção variou de 14 a 16 dias nos animais inoculados com a cepa $Y, 26$ a 29 dias com a Berenice e 9 a 13 dias com a costalimai. O período prepatente variou de 3 a 5 dias nos animais inoculados com a cepa $Y$, de 2 a 6 dias com a cepa Berenice e de 6 a 8 dias com a costalimai. As três cepas apresentaram em Z $\mathbf{Z}$. lasiurus, comprometimento nitidamente muscular, provocando reações leves, moderadas e intensas.
\end{abstract}

UNITERMOS: Trypanosoma cruzi. Zygodontomys lasiurus. Infecção experimental.

\section{INTRODUÇÃO}

Zygodontomys lasiurus é um roedor de ampla distribuição geográfica no Brasil (Mello ${ }^{14}, 1969 ;$ Mello e Moojen ${ }^{19}, 1979$ e Mello ${ }^{15}$, 1980), que se adapta bem em condições de laboratório (Mello e Cavalcante ${ }^{17}, 1982$ ).

Vários autores (Mello e Coelho 18, 1968; Barbosa ${ }^{3}$, 1972; Almeida 1, 1973 e Ri- beiro 22, 1973), verificaram neste roedor a presença de infecção natural por Trypanosoma cruzi, Schistosoma mansoni e Yersinia pestis.

Neste trabalho procurou-se estudar a infecção experimental em $Z$. lasiurus com cepas de $T$. cruzi. A pesquisa teve por objetivo fornecer subsídios que permitam a compreensão do comportamento deste parasita em uma espécie de roedor silvestre.

* Trabalho conduzido com auxílio financeiro do CNPa (Processo: 02-1-06).

* Bolsista do CNPq

*** Da Universidade Federal de São Carlos - Via Washington Luiz - Km 235 - $13560-$ São Carlos, SP - Brasil.

*** Da Universidade de Brasilia - 70910 - Brasília, DF $\rightarrow$ Brasil.

*** Estagiário do Laboratório de Parasitologia - Núcleo de Medicina Tropical - Universidade de Brasília - 70910 - Brasília, DF - Brasil. 
BORGES, M.M. et al. Estudo experimental de Zygodontomys lasiurus (Rodentia-cricetidae) com cepas de Trypanosoma cruzi. Rev. Saúde públ., S. Paulo, 17:387-93, 1983.

\section{MATERIAL E METODOS}

1. Origem da colônia de $Z$. lasiurus e das cepas de $T$. cruzi.

$Z$. lasiurus, utilizado neste trabalho, foi proveniente de uma colônia estabelecida e mantida em laboratório por Mello e Cavalcante ${ }^{1 \tau}$ (1982).

Foram usados dois grupos de animais: 30 adultos com idade de 74 dias e peso variando em torno de $40 \mathrm{~g} ; 15$ jovens-adultos com 30 dias e peso variando em torno de $29 \mathrm{~g}$.

As cepas de $T$. cruzi utilizadas nos experimentos foram: $Y$ e Berenice isoladas de casos humanos (Silva e Nussenzweig ${ }^{25}$, 1953 e Salgado e col. ${ }^{24}$ 1962), e costalimai isolada de triatomíneo silvestre (Mello e Borges ${ }^{16}, 1981$ ).

2. Estudo da evolução da infecção experimental.

Três grupos de 10 animais adultos e 5 jovens-adultos foram inoculados respectivamente por via intraperitonial com: 120.000 tripomastigotas sanguícolas, das cepas humanas, obtidas por punção cardíaca de camundongos infectados; 10.000 tripomastigotas metacíclicos, obtidos da cepa silvestre, mantida "in vitro" em meio LIT.

A contagem dos tripomastigotas sanguicolas foi realizada segundo método de Brener ${ }^{6}$ (1961) e a dos metacíclicos obtidos no LIT, em Câmara de Neubauer (Camargo 8,1964$)$.

A evolução da infecção, nos roedores inoculados, foi acompanhada por 60 dias.

Os exames para determinar a prepatência eram realizados diariamente. Após o periodo prepatente, com o objetivo de seguir a evolução da parasitemia, estes exames passaram a ser realizados em dias alternados.

Os animais que não apresentaram parasitemia patente eram submetidos a xenodiagnóstico com ninfas de II e III estágios de Panstrongylus megistus e Rhodnius neglectus. Os exames destes barbeiros eram realizados após 30 dias do repasto sanguíneo.

A letalidade, ou qualquer outra alteração, era verificada diariamente.
Estudos histopatológicos foram realizados em animais adultos sacrificados após 60 dias de inoculação. Destes animais foram retirados fragmentos dos seguintes órgãos: cérebro, coração, pulmão, esôfago, fígado, baço, intestino delgado e grosso, rim e músculo da coxa. Estes fragmentos foram fixados em formol a $10 \%$ e submetidos a corte histológico, sendo corados com hematoxilina-eosina.

\section{RESULTADOS}

\section{Evolução da Infeç̧ão}

$\mathrm{Na}$ Tabela 1 estão os dados numéricos obtidos sobre a evolução da parasitemia. Observa-se que as cepas Y e Berenice mostraram diferença em relação à idade e $o$ número de parasitas no sangue circulante quando comparadas com a costalimai. Assim é que os animais jovens-adultos sempre apresentaram maior número de parasitas do que os adultos. A cepa $\mathrm{Y}$ apresentou picos de parasitemia no grupo de animais adultos (4.655 tripo) e jovens-adultos (11.500 tripo)

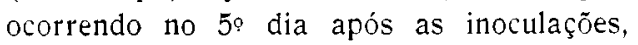
enquanto a cepa Berenice apresentou respectivamente no 6 ? (1.990 tripo) e 10 . dia (8.390 tripo). Os animais inoculados com a cepa costalimai apresentaram número muito baixo de parasitas no sangue circulante. O pico de parasitemia ocorreu, no grupo de animais adultos, no $6^{\circ}$ dia após as inoculações (20 tripo) e nos jovensadultos no 8 : dia (283 tripo). A patência da infecção, no período das observações realizadas, ocorreu como segue: 14 e 12 dias nos animais adultos e jovens-adultos inoculados com a cepa $\mathrm{Y} ; 28$ e 23 dias nos adultos e jovens-adultos inoculados com a cepa Berenice; 4 e 8 dias nos adultos e jovens-adultos inoculados com a cepa costalimai.

$\mathrm{Na}$ Tabela 2 está relatado o resultado sobre o periodo prepatente das três cepas estudadas. Observa-se que houve variações entre os grupos etários inoculados com as cepas humanas. Naqueles inoculados com a cepa $\mathrm{Y}$, estas variações foram de 3-5 dias 
BORGES, M.M. et al. Estudo experimental de Zygodontomys lasiurus (Rodentia-cricetidae) com cepas de Trypanosoma cruzi. Rev. Saúde públ., S. Paulo, 17:387-93, 1983.

T A B E L A 1

Evolução da parasitemia em zygodontomys lasiurus infectado experimentalmente com cepas humanas e sirestres de T. cruzi.

\begin{tabular}{|c|c|c|c|c|c|c|c|}
\hline \multirow{3}{*}{$\begin{array}{r}N^{\top} \\
\text { após }\end{array}$} & \multirow{3}{*}{$\begin{array}{l}\text { de dias } \\
\text { inoculação }\end{array}$} & \multicolumn{6}{|c|}{$\begin{array}{c}\text { Cepas de } T . \text { cruzi } \\
\text { (N: médio de parasitas } / 5 \mathrm{~mm} 3)\end{array}$} \\
\hline & & \multicolumn{2}{|c|}{$\mathrm{Y}$} & \multicolumn{2}{|c|}{$\bar{B}$ erenice } & \multicolumn{2}{|c|}{ Costalimai } \\
\hline & & $\mathrm{AD}$ & JA & $A D$ & $\mathrm{JA}_{\mathrm{A}}$ & $A D$ & $\mathrm{JA}$ \\
\hline & 2 & - & - & 25 & - & - & - \\
\hline & 3 & 185 & 450 & 40 & - & - & - \\
\hline & 4 & $\ldots$ & $\ldots$ & 335 & 90 & - & - \\
\hline & 5 & 4.655 & 11.500 & 625 & $\ldots$ & - & - \\
\hline & 6 & $\ldots$ & $\ldots$ & 1.990 & 5.600 & 20 & 50 \\
\hline & 7 & 470 & 5.200 & $\ldots$ & $\ldots$ & 11 & 40 \\
\hline & 8 & $\ldots$ & $\ldots$ & 1.705 & 3.910 & 5 & 283 \\
\hline & 9 & 220 & 4.730 & $\ldots$ & $\ldots$ & 5 & 190 \\
\hline & 10 & $\ldots$ & 8.933 & $\ldots$ & 8.390 & - & $\ldots$ \\
\hline & 11 & $\ldots$ & $\ldots$ & $\ldots$ & $\ldots$ & $\ldots$ & 80 \\
\hline & 12 & 170 & 2.866 & $\ldots$ & $\ldots$ & - & 10 \\
\hline & 13 & $\ldots$ & $\ldots$ & 135 & 5.200 & - & $\rightarrow$ \\
\hline & 14 & 35 & 33 & $\ldots$ & $\ldots$ & - & - \\
\hline & 15 & $\ldots$ & - & 55 & 1.400 & - & - \\
\hline & 16 & 50 & - & $\ldots$ & $\ldots$ & - & - \\
\hline & 17 & - & - & $\ldots$ & 1.750 & - & - \\
\hline & 18 & - & - & 5 & $\ldots$ & - & -- \\
\hline & 20 & - & - & 50 & 375 & + & - \\
\hline & 21 & - & - & 60 & $\ldots$ & - & -- \\
\hline & 22 & - & - & 20 & 425 & - & - \\
\hline & 24 & - & - & . & 12 & - & - \\
\hline & 25 & - & - & 10 & $\ldots$ & - & - \\
\hline & 26 & - & - & $\ldots$ & 12 & - & - \\
\hline & 27 & - & - & 10 & - & - & - \\
\hline & 29 & - & - & 5 & - & - & - \\
\hline & 30 & - & - & - & - & - & - \\
\hline
\end{tabular}

$\mathrm{AD}=$ Adultos

JA $=$ Jovens-adultos

$+=$ Aniaml encontrado morto

... Exame não realizado

$\rightarrow=$ Resultados negativos

para os adultos e 3 dias para os jovens-adultos, enquanto com a cepa Berenice foram de 2-4 e 4-6 dias para os respectivos grupos. An contrário, nos animais inoculados com a cepa costalimai, o periodo prepatente foi o mesmo de 6-8 dias para os dois grupos etários.

Os xenodiagnósticos realizados nos animais sem parasitemia patente deram resultados positivos.

\section{Achados Histopatológicos}

A análise do material procedente de 10 animais inoculados con a cepa $Y$ revelou o que se segue: miocardite variando de focal discreta a difusa em 8 animais; infiltrado inflamatório focal ou difuso discreto no músculo esquelético em 3 animais. Foram identificados ninhos de amastigotas no músculo cardíaco em um individuo e 
BORGES, M.M. et al. Estudo experimental de Zygodontomys lasiurus (Rodentia-cricetidae) com cepas de Trypanosama cruzi. Rev. Saude pübl., S. Paulo, 17:387-93, 1983.

T A B E I A 2

Período prepatente em Zygodontomys lasiurus infectado com cepas de $T$. cruzi.

\begin{tabular}{lcc}
\hline Cepas de & No de animais/periodo prepatente (dias) \\
\cline { 2 - 3 } T. cruzi & $\mathrm{AD}$ & $\mathrm{JA}$ \\
\hline $\mathrm{Y}$ & $9 / 3,1 / 5$ & $5 / 3$ \\
Berenice & $3 / 2,2 / 3,5 / 4$ & $4 / 4,1 / 6$ \\
Costalimai & $2 / 6,2 / 8,6^{*}$ & $1 / 6,1 / 7,2 / 8,1^{*}$ \\
\hline $\mathrm{AD}=$ Adultos & & \\
$\mathrm{JA}=$ Jovens-adultos & & \\
$* \quad=$ Prepatência não detectada &
\end{tabular}

no músculo esquelético em dois individuos, sem estar, no entanto, relacionado a qualquer reação tecidual.

Os 10 animais inoculados com a cepa Berenice apresentaram o seguinte quadro histopatológico: miocardite focal ou difusa variando de discreta a moderada e miosite discreta em 5 e 7 animais, respectivamente.

Dos 10 animais inoculados com a cepa costalimai, apenas 9 foram submetidos a exame histopatológico, cujos resultados foram os seguintes: 8 apresentaram alterações do miocárdio semelhante às descritas para aqueles animais inoculados com a cepa Berenice; 7 apresentaram alterações intensas do músculo esquelético. Os achados mostraram miosite focal e difusa intensa com destruição de fibras musculares estriadas esqueléticas e reação granulomatosa.

Em 25 animais inoculados com as três cepas de $T$. cruzi, observou-se espessamento e congestão dos septos intralveolares devido a presença de células mononucleares, com alguns apresentando inclusive hemorragia intralveolar. $\mathrm{Em} 10$ animais constatou-se também infiltrado inflamatório mononuclear em torno dos espaços porta do fígado.

Não foi detectado qualquer alteração no material de cérebro, esôfago, baço, intestino delgado e grosso e rim.

\section{COMENTARIOS E CONCLUSÕES}

Os resultados obtidos neste trabalho indicam que $Z$. lasiurus, embora se infecte bem com as cepas utilizadas nos experimentos realizados, não apresentou níveis de suscetibilidade como ocorrido em ratos, camundongos e $C$. callosus (Silva e Nussenzweg ${ }^{25}$, 1953; Leme e Collares ${ }^{13}$, 1962; Brener 7, 1965; Carvalheiro e Collares ${ }^{9}$, 1965; Andrade 2, 1974; Chiari 10, 1977; Melo e Brener 21, 1978; Sogayar 26, 1978; Corsini e col. ${ }^{11}$, 1980; Mello e Borges ${ }^{16}$, 1981 e Borges e col.5, 1982).

As cepas de origem humana, $Y$ e Berenice, são tidas como virulentas para ratos $e$ camundongos albinos. No entanto, quando estas cepas foram inoculadas em $Z$. lasiurus não apresentaram a mesma agressividade, seja esta traduzida pela parasitemia ou pelos achados histopatológicos. Trinta por cento dos 2 . lasiurus inoculados com a cepa Y e $70 \%$ com a cepa Berenice apresentaram graus discretos de envolvimento muscular esqulético.

Comparando também os resultados deste trabalho com aqueles realizados em $C$. callosus (Borges e col. ${ }^{5}, 1982$ ), em que foi utilizada metodologia semelhante, i.e., inoculações por via intraperitoneal e doses de 120.000 tripomastigotas sanguícolas de camundongos, verifica-se que $Z$. lasiurus apresenta niveis de parasitemia bem mais baixos do que aquela espécie, embora a patência e prepatência tenham sido semethantes, assim como o tropismo muscular.

Em relação a cepa costalimai, $Z$. lasiurus também apresentou niveis de parasitemia e patência inferiores àqueles obtidos em $C$. 
BORGES, M.M. et al. Estudo experimental de zygodontomys lasiurus (Rodentia-cricetidae) com cepas de Trypanosoma cruzi. Rev. Saúde públ., S. Paulo, 17:387-93, 1983.

callosus e camundongos (Mello e Borges ${ }^{16}$, 1981). Em relação ao comprometimento muscular, $77 \%$ dos $Z$. lasiurus, apresentaram graus de reação que variaram de moderado a severo.

Poucos estudos têm sido conduzidos sobre infeç̧ão experimental de mamiferos silvestres com $T$. cruzi (Spinoza12, 1953; Ryckman e Robb 23, 1965; Mello e col.20, 1979; Borges e Mello 4, 1980; Mello e Borges 16, 1981 e Borges e col.5, 1982). Não obstante ser a doença de Chagas autoctone do continente Americano, excetuando cobaias, animais utilizados como modelo experimental desta parasitose (ratos, camundongos, hamsters, coelhos e cães) são todos do velho mundo. Os roedores compreendem um grupo de mamíferos com numerosas espécies envolvidas no ciclo silvestre de $T$. cruzi. Estudos experimentais com estes animais sem dúvida fornecem subsidios à compreensão de vários aspectos da epizootologia do $T$. cruzi, nos focos naturais, como mostraram os trabalhos de Ryckman e Robb ${ }^{23}$ (1965). Estes autores realizaram estudos sobre infecção experi- mental de $T$. cruzi em Neotoma fuscipes e $N$. lepida, com o objetivo de verificar se estes roedores seriam capazes de infectar triatomíneos com este protozoário por longos períodos. Os resultados obtidos explicaram, em parte, diferenças nos aspectos da dinâmica de transmissão no que se relacionava ao papel que cabia a cada espécie na circulação de $T$. cruzi.

A espécie de roedor aqui estudada, $Z$. lasiurus, que se adapta bem em condições de cativeiro (Mello e Cavalcante ${ }^{17}$, 1982) e que já foi encontrada naturalmente infectada por T. cruzi (Mello e Coelho 18, 1968; Ribeiro 22, 1973), poderia, à exemplo de $C$. callosus (Mello e col.20, 1979; Borges e Mello 4, 1980; Mello e Borges ${ }^{16}, 1981$ e Borges e col. ${ }^{5}, 1982$ ) ser também utilizada em pesquisas experimentais com este protozoário. Observações em laboratório poderiam dar também contribuições que pudessem ser relacionadas as observações de campo, no sentido de vir oferecer base para elucidar hipóteses em relação aos mecanismos de transmissão em mamiferos silvestres.

BORGES, M. M. et al. [Studies on the experimental infection of Zygodontomys lasiurus (Rodentia-Cricetidae) with three strains of Trypanosoma cruzi]. Rev. Saúde públ., S. Paulo, 17:387-93, 1983.

ABSTRACT: Studies on the experimental infection of Zygodontomys lasiurus (Rodentia-Cricetidae) are presented in the current paper. Three strains of $\mathbf{T}$. cruzi were used in the experiments: two, $Y$ and Berenice, were originated from human infection, while the third one was obtained from natural infection in a wild bug Triatoma costalimai. The evolution of the parasitemia was studied and the prepatent and patent periods determined. The tissular tropism and aggressiveness of the strains of $\mathbf{T}$. cruzi were verified through histopathological studies. Results have shown that the parasitemia was always kept at low levels for all the strains. The prepatent period in the infected rodents varied from: 3 to 6 days in the $\mathrm{Y}$ strain; 2 to 6 days in the Berenice strain; 6 to 8 in the costalimai strain. The patent period showed the following duration patterns: 14 to 16 days in the $\mathrm{Y}$ strain; 26 to 29 in the Berenice strain and 6 to 8 days in the costalimai strain. Tissue damage was predominantly muscular, and the pathological reactions varied from slight to severe.

UNITERMS: Trypanossoma cruzi. Zygodontomys lasiurus. Experimental infection. 
BORGES, M.M. et al. Estudo experimental de Zygodontomys lasiurus (Rodentia-cricetidae) com cepas de Trypanosoma cruzi. Rev. Saude publ., S. Paulo, 17:387-93, 1983.

\section{REFERENCIAS BIBLIOGRAFICAS}

1. ALMEIDA, C.R. Relatorio das pesquisas do plano piloto de peste em Exu. Salvador, Centro de Pesquisas Aggeu Magalhães. 1973.

2. ANDRADE, S. Caracterização de cepas de T. cruzi isoladas no Recôncavo Baiano. Rev. Pat. trop., 1:65-121, 1974.

3. BARBOSA, F.S. Natural infection with Schistosoma mansoni in small mammals trapped in the course of a schistosomiasis control project in Brazil. $J$. Parasit., 58:364-365, 1972.

4. BORGES, M.M. \& MELLO, D.A. Infectividade de cepas silvestres de Trypanosoma cruzi mantidas em cultura, para Calomys callosus (Rodentia) e camundongos albinos. Rev. Pat. trop., 9:145$161,1980$.

5. BORGES, M.M.; MELLO, D.A. \& TEIXEIRA, M.L. Infecção experimental de Calomys callosus (Rodentia-Cricetidae) com Trypanosoma cruzi. Rev. Saúde públ., S. Paulo, 16:233-42, 1982.

6. BRENER, Z. Contribuição ao estudo da terapêtica experimental da Doença de Chagas. Belo Horizonte, 1961. [Tese de Livre Docência - Faculdade de Odontologia e Farmácia da UFMG].

7. BRENER, Z. Comparative studies of different strains of Trypanosoma cruzi. Ann. trop. Med. Parasit., 59:19-26, 1965.

8. CAMARGO, E.P. Growth and differentiation in Trypanosoma cruzi. I. Origin of matacyclic trypanosoma in liquid media. Rev. Inst. Med. trop. S. Paulo, 6:93-100, 1964.

9. CARVAlHeiro, J.R. \& COLlares, E.F. Estudos sobre o comportamento em cam:ndongos de uma amostra altamente virulenta de Trypanosoma cruzi (Amostra $Y)$ após passagem em triatomineos, ratos e culturas. Rev. bras. Biol., $25: 169-75,1965$.

10. CHIARI, C.A. Comportamento de diferentes amostras de Trypanosoma cruzi em cobaias experimentalmente infectadas. In: Reunião Anual de Pesquisa Básica em Doença de Chagas, Caxambú, 1977. Caxambú, 1977. p. 23, [resumo]
11. CORSINI, A.C.; COSTA, M.G.; OLIVEIRA, L.P. CAMARGO, I.J.B. \& STELINI Jr., A. Susceptibility of inbred mice to Trypanosoma cruzi strain Y. Rev. Inst. Med. trop. S. Paulo, 22:192-6, 1980.

12. ESPINOZA, L.A. Algunas consideraciones sobre el comportamiento del Trypanosoma cruzi (Schizotrypanum cruzi en el Didelphis azarae o Didelphis paraguayensis (Zarugueyas, zorros comun a raposa). Rev. ecuat. Hig. Med. trop., $10: 27-34,1953$.

13. LEME, M.V.S. \& COLLARES, E. F. Estudos sobre a suscetibilidade de ratos brancos a uma amostra de Trypanosoma cruzi altamente virulenta para camundongo (Amostra Y). Med. Rev. CARL, 1:109$-11,1962$.

14. MELLO, D.A. Roedores silvestres de alguns municípios do Estado de Pernambuco e suas regiōes naturais. Rev. bras. Pesq. méd. biol., 2:360-2, 1969.

15. MELLO, D.A. Estudo populacional de algumas espécies de roedores do Cerrado (norte do municipio de Formosa, Goiás). Rev. bras. Biol., 40:843-60, 1980.

16. MELLO, D.A. \& BORGES, M.M. Primeiro encontro do Triatoma costalimai naturalmente infectado pelo Trypanosoma cruzi: estudo de aspectos biológicos da amostra isolada. Mem. Inst. Oswaldo Cruz, $76: 61-9,1981$.

17. MELLO, D.A. \& CAVALCANTE, J.P. Estudo do ciclo biológico do Zygodontomys lasiurus (Cricetidae), em conđições de laboratório. Bras. flor. 1982. (no prelo)

18. MELLO, D.A. \& COELHO, A.S.M. Sobre tripanossomas de roedores silvestres da regiāo do Araripe do Estado de Pernambuco. Rev. Soc. bras. Med. trop., 2:67-70, 1968.

19. MELLO, D.A. \& MOOJEN, L.E. Nota sobre uma coleção de roedores e marsupiais de algumas regiões do Cerrado do Brasil Central. Rev. bras. Pesq. med. biol, 18:287-91, 1979. 
BORGES, M.M. et al. Estudo experimental de Zygodontomys lasiurus (Rodentia-cricetidae) com cepas de Trypanosoma cruzi. Rev. Saúde públ., S. Paulo, 17:387-93, 1983.

20. MELLO, D.A.; VALIN, E. \& TEIXEIRA, M.L. Alguns aspectos do comportamento de cepas silvestres de Trypanosoma cruzi em camundongos e Calomys callosus (Rodentia). Rev. Saúde públ., S. Paulo, 13:314-25, 1979.

21. MELO, R.C. \& BRENER, Z. Tissue tropism of different Trypanosoma cruzi strain. J. Parasitol., 64:475-82, 1978.

22. RIBEIRO, R.D. Novos reservatórios do Trypanosoma cruzi. Rev. bras. Biol., $33: 429-37,1973$.

23. RYCKMAN, R.E. \& ROBB, P.L. Epizootiology of Trypanosoma cruzi in southwestern North America. Part III. Epizootiological and pathological histories of Trypanosoma cruzi infections in natural reservoir hosts (Kinetoplastida: Trypanosomidae) (Rodentia: Cricetidae) (Hemiptera: Triatominae). J. med. Entomol., $2: 91-3,1965$.
24. SALGADO, J.A.; GARCEZ, P.N.; OLIVEIRA, C.A. \& GALIZZI, J. Revisão clinica atual do primeiro caso humano descrito da Doença de Chagas. Rev. Inst. Med. trop. S. Paulo, 4:330-7, 1962.

25. SILVA, L.H. \& NUSSENZWEIG, V. Sobre uma cepa de Trypanosoma oruzi altamente virulenta para camindongo branco. Folia clin. biol., 20:191-207, 1953.

26. SOGAYAR, R. Infecção experimental de ratos albinos "wistar" com diferentes cepas de Trypanosoma cruzi, Chagas 1900. Belo Horizonte, 1978. [Dissertação de Mestrado - Dept. Parasitologia ICB da Universidade Federal de Minas Gerais].

Recebido para publicagão em 09/02/1983 Aprovado para publicação em 03/06/1983 